\title{
Temporalis Muscle Flap for the Immediate Reconstruction of Oral Defects after Oncologic Resection
}

\author{
Hong Loi Nguyen ${ }^{a} \quad$ Van Minh Nguyen ${ }^{b} \quad$ Xuan Phu Tran ${ }^{a}$ \\ ${ }^{a}$ Odonto-Stomatology Center, Hue Central Hospital, Hue, Vietnam; bFaculty of \\ Odonto-Stomatology, Hue University of Medicine and Pharmacy, Hue, Vietnam
}

\section{Keywords}

Squamous cell carcinoma - Maxillary defect $\cdot$ Reconstruction $\cdot$ Temporalis muscle flap

\begin{abstract}
An oral defect after oncologic resection, especially in the upper jaw, is an extremely complex problem in terms of reconstructive surgery. Herein, we report 2 patients who were diagnosed with oral mucosal squamous cell carcinoma. In one patient, the lesion was present on the right side of the hard palate, invading the maxillary bone. In the other patient, the lesion was present in the left retromolar trigone region. Reconstruction of the postsurgical defects was successfully done using a temporalis muscle flap. Both patients were discharged after ensuring the absence of any postsurgical complications. Furthermore, the postoperative functional and esthetic outcomes were adequate. These case reports highlight the usefulness and reliability of a temporalis muscle flap for the immediate reconstruction of oral and maxillary defects after oncologic resection.

\section{Introduction}

Cancer is one of the main causes of death, and its rate of occurrence has increased along with the diversity in morphological types. Oral cavity carcinoma is one of the eight most prevalent types of cancer in the world. It occurs due to a malignant change in the oral mucous membrane. Squamous cell carcinoma is the most common malignant tumor of the oral cavity $[1,2]$. 
Fig. 1. A mucosal ulcer lesion in the left retromolar trigone region.

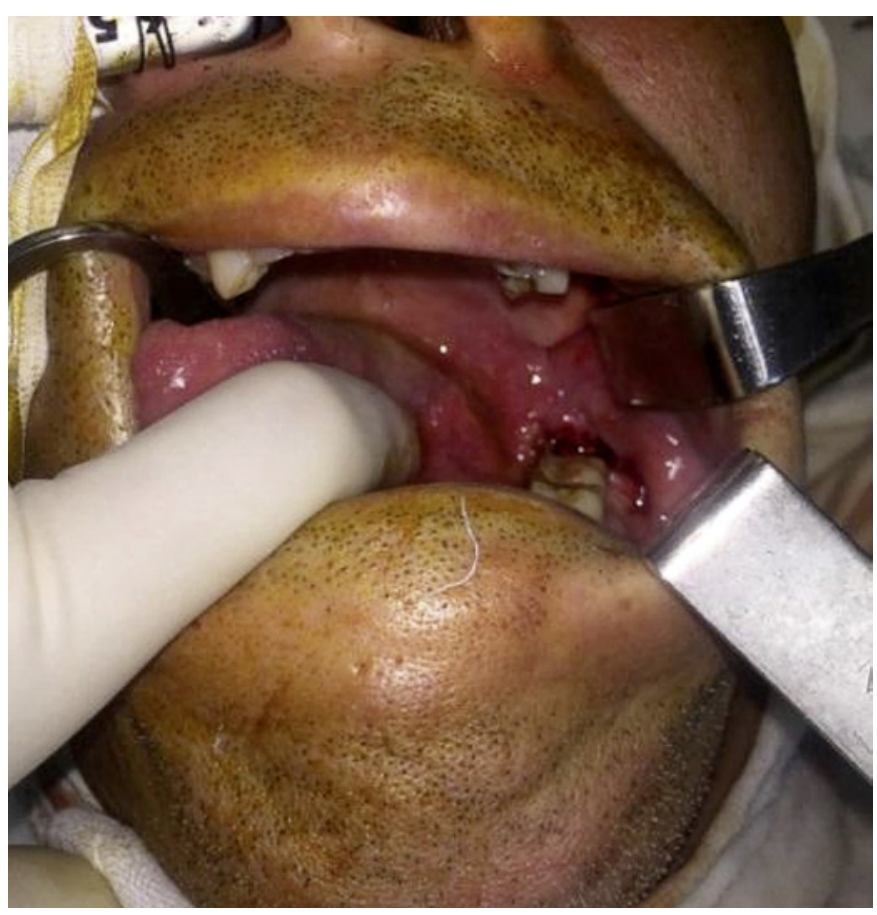

The orofacial region is involved in several functions, such as mastication, speech, deglutition, and lifting of the eyelids. It also plays an important role in esthetics. These functions can be attributed to the presence of unique anatomical components, such as the eyes, nose, and mouth. An oral defect after oncologic resection, especially in the upper jaw, is an extremely complex problem in terms of reconstructive surgery. The techniques used to reconstruct this defect include local flaps, regional flaps, and microsurgical flaps. The temporalis muscle flap (TMF) in particular is a regional flap that is often used to reconstruct oral defects, especially after oncologic resection of the maxillary bone and retromolar trigone region [3-5].

Herein, we report on the surgical technique and early outcomes of the TMF used for immediate reconstruction in 2 cases of oral defect after oncologic resection.

\section{Case Report}

Case 1

A 56-year-old man reported to the Center of Odonto-Stomatology, Hue Central Hospital, with a complaint of ulceration on the left side of the lower jaw. Lesions had appeared about 2 months prior and caused difficulty in eating and drinking. An intraoral examination revealed mucosal ulcer lesions measuring $2 \times 2 \mathrm{~cm}$. The lesions demonstrated easy bleeding on palpation (Fig. 1). Physical examination did not reveal any cervical lymphadenopathy. Histopathological findings were suggestive of well-differentiated squamous cell carcinoma. A computed tomography (CT) scan of the head and neck area showed that the lesion had not metastasized to the lower jaw. However, few cervical lymph nodes measured $1.5 \mathrm{~cm}$.

The patient was diagnosed with stage T2N0M0 squamous cell carcinoma of the left retromolar trigone. The tumor was managed by oncologic resection followed by immediate reconstruction using a TMF and selective neck dissection (removal of lymph nodes at levels I-III) (Fig. 2).

\section{Karger' ${ }^{\prime \prime}$}




\section{Case Reports in Oncology}

\begin{tabular}{l|l}
\hline Case Rep Oncol 2021;14:573-579 \\
\hline DOI: 10.1159/000514630 & $\begin{array}{l}\text { ○ 2021 The Author(s). Published by S. Karger AG, Basel } \\
\text { www.karger.com/cro }\end{array}$ \\
\hline
\end{tabular}

Nguyen et al.: Temporalis Muscle Flap for Maxillary Reconstruction
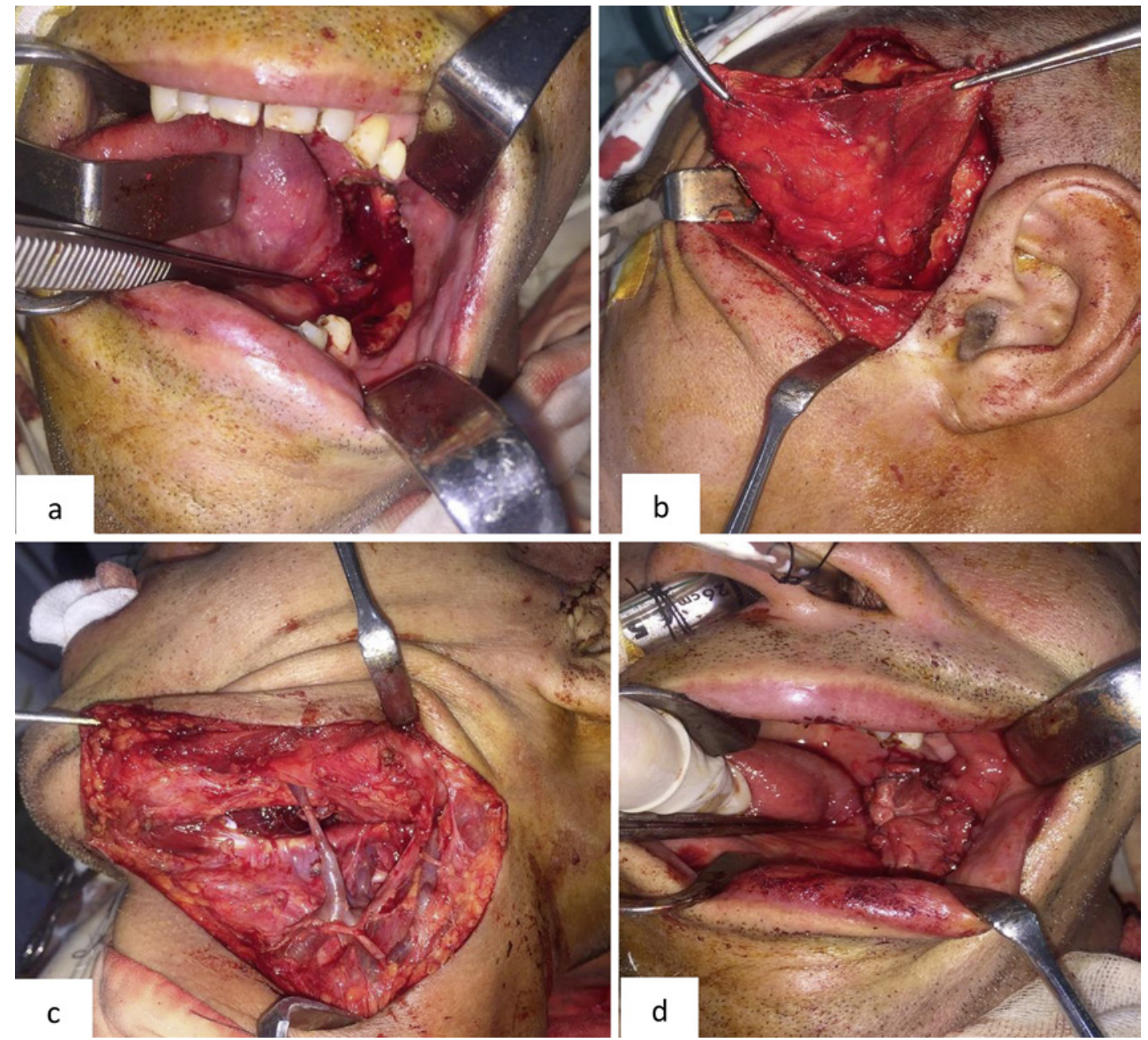

Fig. 2. Surgical procedure. a, b Oncologic resection. c Selective neck dissection. d Immediate reconstruction using a temporalis muscle flap.

Fig. 3. Clinical view of squamous cell carcinoma on the right side of the hard palate invading the maxilla.

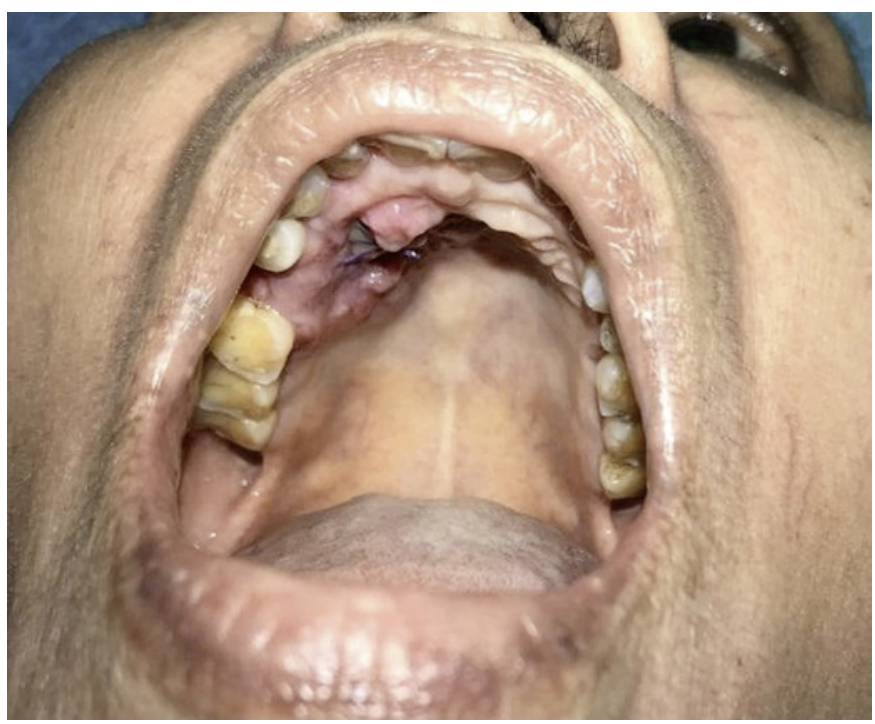




\section{Case Reports in Oncology}

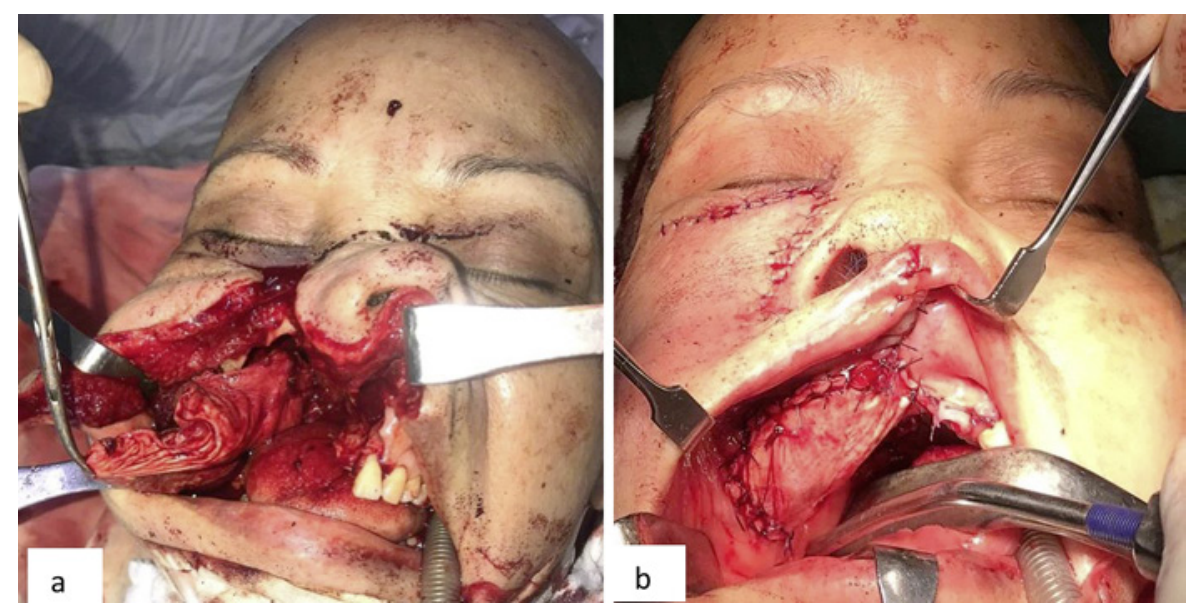

Fig. 4. Surgical procedure. a Maxillectomy on the right side. b Immediate reconstruction using a temporalis muscle flap.

Case 2

A 61-year-old female patient was admitted to the Center of Odonto-Stomatology, Hue Central Hospital, for the treatment of oral mucosal lesions. A detailed case history revealed that the ulcerative lesions on the right side of the hard palate had developed 4 months previously. The patient complained of frequent bleeding from the lesion site. An intraoral examination revealed mucosal ulcer lesions measuring $2 \times 2 \mathrm{~cm}$ (Fig. 3). The physical examination did not reveal any cervical lymphadenopathy. The histopathological findings of the biopsy specimen were suggestive of well-differentiated squamous cell carcinoma. A CT scan of the head and neck area showed that the lesion had invaded the upper jaw.

Based on the clinical, histopathological, and CT scan findings, it was diagnosed as stage T4aN0M0 squamous cell carcinoma of the right hard palate with maxillary bone invasion. Treatment included oncologic resection of the entire right maxilla and immediate reconstruction using a TMF. According to Brown's classification of maxillectomy, the resultant defect was classified as type 2a (Fig. 4).

\section{Discussion}

The management of maxillary defects after oncologic resection is based on many factors, such as the location of defect and the patient's general health condition. There are several options available for the reconstruction of maxillary defects based on their size.

Obturation is the simplest method for the rehabilitation of maxillectomy defects. However, it has many disadvantages, such as susceptibility to infection due to difficulty in hygiene maintenance and poor fit, which makes it difficult to stabilize.

A microvascularized free flap is not suitable for all maxillectomy patients, especially older patients and patients with cardiovascular risk factors, as they may require postoperative radiotherapy. In these patients, there may be a high risk of failure of the vascular anastomoses and consequent flap loss [6].

The TMF is a reliable and versatile choice that is widely used for reconstruction of intraoral tissue defects following surgical oral cancer therapy. There are several advantages of a TMF: (1) the donor and defect sites are in the same surgical field, which prevents the need for second surgery to acquire the flap from elsewhere; (2) the donor site has sufficient tissue for recon- 

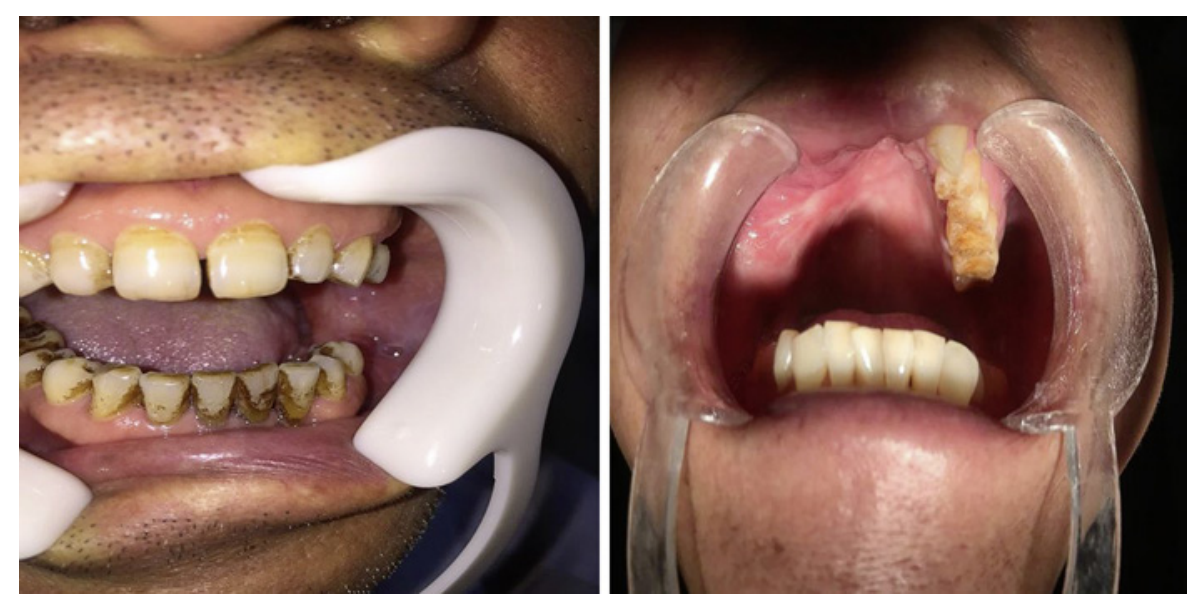

Fig. 5. Clinical view of reepithelialized temporalis muscle flap postoperatively, which had reepithelialized completely after 2 months.

struction; (3) the flap is well vascularized due to dual blood supply; (4) the functional results are acceptable; (5) it is available on both sides of the temporal area; (6) intraoperative complications are minimal; and (7) there is less postoperative damage to the donor site [7]. Both of our patients showed adequate functional recovery 4 months after surgery. These patients had no difficulty in speaking, eating, or swallowing. Ocular function was also unaffected.

Most authors agree that the reconstruction procedure should be accomplished during the same surgical operation, immediately following oncologic resection. In the present cases, an acceptable quality of life was restored for both patients 2 months after the operation. Both patients were satisfied with their postoperative facial appearance, speech, and ability to swallow and masticate. Intraoral examination after 4-6 weeks revealed a reepithelialized TMF (Fig. 5). Three months later, the color and texture of the myofascial flap was similar to that of the adjacent oral mucosa [6,8].

The main disadvantage of the TMF is the development of a concave defect in the temporal fossa region, which was seen in both of our patients. The patient in case 2 , who underwent right maxillectomy, developed facial asymmetry due to the loss of bone support. To overcome this problem, a partial denture is indicated to improve esthetics and restore mastication. Alternatively, some authors recommend silicone fillers to augment the temporal fossa defect, but this involves a risk of infection. This cosmetic problem can also be resolved by letting the hair grow long enough to cover the defect.

Early complications of the TMF technique are hematoma, infection of the temporal fossa, and partial or total flap necrosis. The incidence of partial flap loss and temporal branch paresis is reported to be 13 and $19 \%$, respectively [6, 7]. In case 1, the patient suffered from postoperative infection of the temporal fossa due to an upstream infection from the oral cavity. This complication was managed by performing pus drainage, suturing of the oral fistula, and antibiotic therapy. After 2 weeks, the infection subsided and the patient's condition was stable. The flap was still viable without any evidence of necrosis.

Rapidis et al. [9] reported that the risk of partial dehiscence of a TMF is high if the defect after maxillectomy is more than $4 \times 4 \times 3 \mathrm{~cm}$ in size. However, some authors reported that the TMF can close a maxillary defect measuring up to $6 \times 4 \mathrm{~cm}$. In case 2 , the defect was $3 \times$ $3 \times 2 \mathrm{~cm}$ in size. Follow-up after 4 months revealed no evidence of TMF dehiscence $[6,10]$.

The main objective of any reconstructive surgery is to restore esthetics and function. However, the TMF does not restore the jawbone defect. In case 2, due to the loss of the entire

\section{Karger'}




\section{Case Reports in Oncology}
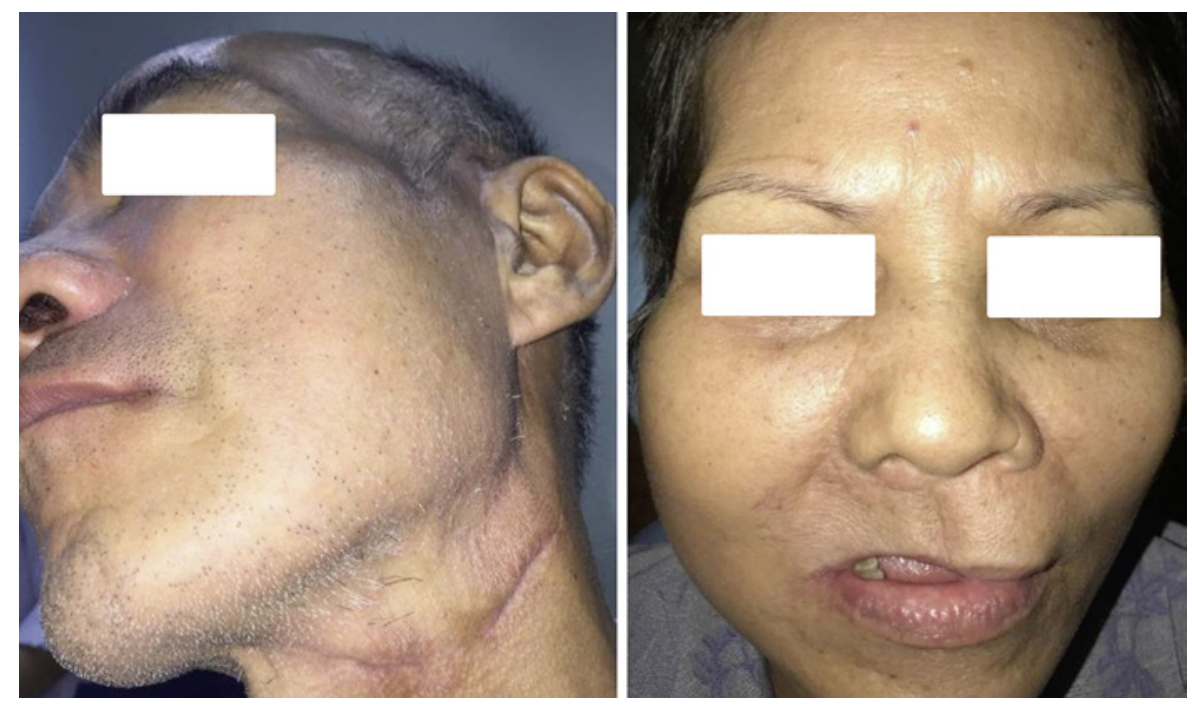

Fig. 6. Postoperative esthetic defects.

right maxillary bone (type 2a), the elevation of the right side of the upper lip was insufficient, which led to an esthetic defect (Fig. 6). Therefore, it is necessary to have a prosthodontic treatment plan to restore the mastication ability and esthetics.

In conclusion, the use of a TMF for the reconstruction of postoperative defects of oral mucosal cancer is a safe and reliable technique. The esthetic and functional outcomes are good and there is less damage to the donor site. Furthermore, the postoperative quality of life is acceptable to the patient.

\section{Statement of Ethics}

The patients have given their written informed consent for the publication of their case including images.

\section{Conflict of Interest Statement}

The authors declare no conflict of interest in association with this article.

\section{Funding Sources}

There were no funding sources.

\section{Author Contributions}

All individuals who qualify as authors have been listed; each has participated in the conception and design of this work, the writing of the document, and the approval of the submission of this version. 


\section{References}

1 Hassanein AG. Continuous validity of temporalis muscle flap in reconstruction of postablative palatomaxillary defects. J Craniofac Surg. 2017 Mar;28(2):e130-7.

2 Krzymański G, Dąbrowski J, Przybysz J, Domański W, Biernacka B, Piętka T. Temporal muscle flap in reconstruction of maxillo-facial tissues. Contemp Oncol (Pozn). 2012;16(3):244-9.

3 Clauser L, Curioni C, Spanio S. The use of the temporalis muscle flap in facial and craniofacial reconstructive surgery. A review of 182 cases. J Craniomaxillofac Surg. 1995;23(4):203-14.

4 Dallan I, Lenzi R, Sellari-Franceschini S, Tschabitscher M, Muscatello L. Temporalis myofascial flap in maxillary reconstruction: anatomical study and clinical application. J Craniomaxillofac Surg. 2009 Mar;37(2):96-101.

5 Spanio di Spilimbergo S, Nordera P, Mardini S, Castiglione G, Chim H, Pinna V, et al. Pedicled temporalis muscle flap for craniofacial reconstruction: a 35-year clinical experience with 366 flaps. Plast Reconstr Surg. 2017 Feb;139(2):468e-76e.

6 Ahmed Djae K, Li Z, Li ZB. Temporalis muscle flap for immediate reconstruction of maxillary defects: review of 39 cases. Int J Oral Maxillofac Surg. 2011 Jul;40(7):715-21.

7 Pires FR, Ramos AB, Oliveira JB, Tavares AS, Luz PS, Santos TC. Oral squamous cell carcinoma: clinicopathological features from 346 cases from a single oral pathology service during an 8-year period. J Appl Oral Sci. 2013 Sep-Oct;21(5):460-7.

8 Feller L. Oral squamous cell carcinoma: epidemiology. clinical presentation and treatment. J Cancer Ther. 2012;3(4):263-8.

9 Rapidis AD, Alexandridis CA, Eleftheriadis E, Angelopoulos AP. The use of the buccal fat pad for reconstruction of oral defects: review of the literature and report of 15 cases. J Oral Maxillofac Surg. 2000;58(2):158-63.

10 Browne JD, Butler S, Rees C. Functional outcomes and suitability of the temporalis myofascial flap for palatal and maxillary reconstruction after oncologic resection. Laryngoscope. 2011 Jun;121(6):1149-59. 